\title{
Applying Linear Wavelet Transforms and Statistical feature Analysis for Digital Tongue Image
}

\author{
M. Dhanalakshmi ${ }^{1}$, P. Premchand ${ }^{2}$, A.Goverdhan ${ }^{3}$ \\ ${ }^{I}$ Department of Computer science, CEJ- JNTUH University, India \\ ${ }^{2}$ Department of Computer science, Osmania University, India \\ ${ }^{3}$ Department of Computer science, SIT-JNTUH University, India
}

\begin{abstract}
The present paper proposes a novel scheme of application of linear wavelets in Digital image processing and statistical evaluation of tongue image for diagnosis in Ayurvedic medical system. The proposed method attempts to differentiate the tongue of a person with healthy or unhealthy conditions. It consists of two techniques applied sequentially: first, enhancement of the tongue image for extracting the true nature of colors and textures on different parts of the tongue and applying wavelets transform; second, computing the statistical features to identify, whether the tongue image belongs to a healthy or unhealthy person. The scheme is implemented on a database including images from internet and selected college hostel students. The analysis of the results reveals that our method can significantly assist in the tongue diagnosis. This proposed method helps in expanding the scope of tongue diagnosis system in Ayurvedic medical system towards reliable and computationally inexpensive diagnostic model.
\end{abstract}

Keywords : Ayurvedic Tongue diagnosis, Digital Image Processing, Wavelets, Statistical Features.

\section{INTRODUCTION}

The Traditional Medical practitioners rely on patient's bodies to tell them whether something is wrong in the patient's body. Different visible parts of the body like the skin, hair, eyes, lips and nails as indicators of internal imbalance and the tongue can also provide them with vital clues [1]. In fact, in traditional Chinese and Indian Ayurvedic medicine, observation of the tongue plays a fundamental role in determining a person's status of health. This system of diagnosis dates back to several thousand years, and has long been used as an investigative tool by practitioners of traditional schools of medicine. Modern Western medicine recognizes that the tongue can be a mirror of illnesses which are systemic and not just local to the tongue. Traditional Indian medicine-Ayurveda has developed the observation of the tongue into a general system of diagnosis.

In Ayurvedic medicine the tongue is seen to not only shows the symptoms of diseases but also to reflect the degree of health or imbalance of the body. The tongue is examined to assist in diagnosis and to the extent in which the tongue changes with treatment can indicate how successful the treatment has been [2]. 'Tongue analysis' is one of the processes in the Ayurvedic system [3] of patient understanding and management. Ayurvedic practitioners ask the patient to open their mouth and show the tongue to extract the required visual information. The practitioner's interest in the tongue are -1) size and shape of the tongue 2)tip 3)width 4)thickness 5) color 6)texture 7) projections 8) fissures \& cuts 9) coating/fur 10) swelling 11)bleeding 12) and above conditions in different parts of the tongue, etc. Based on this visual information and with their knowledge and experience; Medical practitioners try to understand the condition of the patient. The role of digital image processing comes as a tool developer to the analysis of tongue image. It is better if we examine and understand the color feature and texture feature of the tongue image of a healthy person and unhealthy person separately to apply the computer aided techniques for the evaluation of the tongue. The processing of tongue image navigates through different steps like: enhancement of image, identifying the color and texture of the tongue, highlighting the irregularities and application of statistical differentiators etc...

There were many attempts to implement Digital image processing techniques for the processing of the tongue image to make it more useful for medical practitioners. A lot of research was done for traditional Chinese medicine. Recently, new approaches for processing the tongue images have received a great deal of interest among researchers. A brief review of some of the recent researches is presented here. Wangmeng Zuo et al. [4] presented a technique for tongue image segmentation by merging polar edge detector and active contour model. The results revealed that the tongue segmentation can segment the tongue precisely. A measurable assessment on 50 images shows that the mean DCP (the distance to the closest point) of the proposed technique is 5.86 pixels, and the average true positive (TP) percent is $97.2 \%$. Bo Pang et al. [5] presented a tonguecomputing model for the diagnosis of appendicitis based on quantitative measurements that comprise chromatic and textural metrics. Customary tongue diagnosis is always dedicated to the identification of syndromes other than ailments. Applying their method to clinical tongue images, the tentative results are promising. Yang Ben Sheng et al. [6] proposed an image segmentation algorithm centered on the shortest path. The algorithm is 
superior to the conventional region growing algorithm, to overcome certain disadvantages experienced by oldfashioned region growing built on competing seeds. Algorithm depicts the contours of the object area precisely, particularly when it is used to segment the local object of the image, the segmentation outcomes are useful to sequence image analysis and patter recognition. Wang X and Zhang D [7] proposed an optimized correction scheme that amends the tongue images captured in various device-dependent color spaces to the target deviceindependent color space. While the tentative results on real tongue images illustrate that the distorted tongue images (taken in different device-dependent color spaces) become steadier with each other. In actual fact, the average color difference amid them is significantly abridged by more than $95 \%$. The segmentation of the body of tongue plays a significant part in automatic tongue diagnosis in Traditional Chinese Medicine. If there are comparable grayscales near the boundaries of the body of tongue, it is tough to excerpt the body of tongue suitably with some standard methods directly. In order to overcome this effort, Wenshu Li et al. [8] have offered a technique that joins prior knowledge with improved.

As explained above, several methods $[9,10,11,12,13,14,15]$ have been proposed for the analysis of tongue image segmentation and every method performed good by its own algorithm and function. Though researchers have made significant advancement in the standardization and quantification of tongue diagnosis, there are still significant problems with the existing approaches. First, most of the methods are only concerned with the detection of special features on tongue and their separation from the original image. Second, the original validity of these methods and systems is usually derived from a comparison between the diagnostic results that are acquired from the methods or systems and the judgments made by skillful practitioners of tongue diagnosis. Third, only few samples are used in the experiments and this is far from meeting the requirements of obtaining a reasonable result in statistical pattern recognition. Based on the above literature survey the present paper proposes a new one-level linear wavelet decomposition technique of Tongue analysis based on texture features derived from co-occurrence parameters and application of statistical features on the image to get a valid result on the health condition of the person of that image. To validate the proposed method, JNTUH-students database images and Google images are considered for the experimental analysis.

The remainder of the paper is organized as follows: section (2) introduction to wavelets, section (3) focuses on proposed Tongue analysis approach, section (4) describes results and discussion and section (5) describes the conclusion.

\section{INTRODUCTION TO WAVELETS}

The wavelet transform is a multi-resolution technique, that can be implemented as a tree structure and is similar to sub-band decomposition[16,17].There are various wavelet transforms like Haar, Daubechies(Db6), Coiflet (Cf6), Symlet (Sym8) and etc. They differ with each other in the formation and in the reconstruction. The wavelet transform divides the original image into four sub bands and they are denoted by LL (low-low), LH (low-high), HL (high-low) and HH (high-high) frequency sub bands. The HH sub image represents diagonal details (high frequencies in both directions), HL gives horizontal high frequencies (vertical edges), LH gives vertical high frequencies (horizontal edges), and the image LL corresponds to the lowest frequencies. At the subsequent scale of analysis, the image LL undergoes the decomposition using the same filters, having always the lowest frequency component located in the upper left corner of the image. Each stage of the analysis produces next four sub images whose size is reduced twice when compared to the previous scale. i.e. for level ' $n$ ' we get a total of ' $4+(n-1)^{*} 3$ ' sub bands. The size of the wavelet representation is the same as the size of the original. The Haar wavelet method [17] is the first known wavelet and was proposed by Alfred Haar. He used these functions to give an example of a countable orthonormal system for the space of square-integral functions on the real line. The Haar wavelet's scaling function coefficients are $h\{k\}=\{0.5,0.5\}$ and wavelet function coefficients are $\mathrm{g}\{\mathrm{k}\}=\{0.5,-0.5\}$. The Daubechies wavelets [18] are a family of orthogonal wavelets defining a discrete wavelet transform and characterized by a maximal number of vanishing moments for some given support. With each wavelet type of this class, there is a scaling function which generates an orthogonal multiresolution analysis.

\section{Proposed Methodology}

In the first stage of the proposed methodology, the several types of filtering and enhancement techniques are tried to improve the image quality of the tongue as in most of the cases, the direct image from the camera is not found to be suitable for the extraction of the statistical features for the differentiation from the healthy and unhealthy person. After much research on the enhancement, two enhancement techniques were evolved with enhancement threshold factors ETF-1 and ETF-2 which are found to give better results for the classification of the images. To overcome the complexity of using statistical features on entire image, the image is decomposed with linear one-level wavelet decomposition technique i.e. Haar and Daubechies. Then the methodology crops the major centre portion of the decomposed part and evaluates the texture features of cropped part. The proposed method crops the tongue image with a standard pixel size of 100x100. The tongue 
statistical features (TSF) are derived from the statistical parameters [19]. They are given in the following definitions and equations.

TSF1: Mean $(f(x, y))$ - it is most basic of all statistical measure. Its mathematical formulation is given as follows

$$
f(x, y)=\frac{1}{m n} \sum_{(r, c) \in W} g(r, c)
$$

TSF2: Standard Deviation $\left(f^{f}(x, y)\right)$-It is a most widely used measure of variability or diversity used in Mathematical representation of standard deviation is given by

$$
\tilde{f}(x, y)=\sqrt{\frac{1}{m n-1} \sum_{(r, c) \in W}\left(g(r, c)-\frac{1}{m n-1} \sum_{(r, c) \in W} g(r, c)\right)^{2}}
$$

TSF3: Entropy - it is the measure of randomness. Its mathematical representation is given by following equation.

$$
\text { Entropy: } \quad-\sum_{i=0}^{G-1} \sum_{j=0}^{G-1} p(i, j) \log _{2}[p(i, j)]
$$

The following figures- 1 and 2 are the examples of the images from the database considered for the evaluation of our proposed technique. The sample original tongue images are shown in below and they are named from (a) to (1).

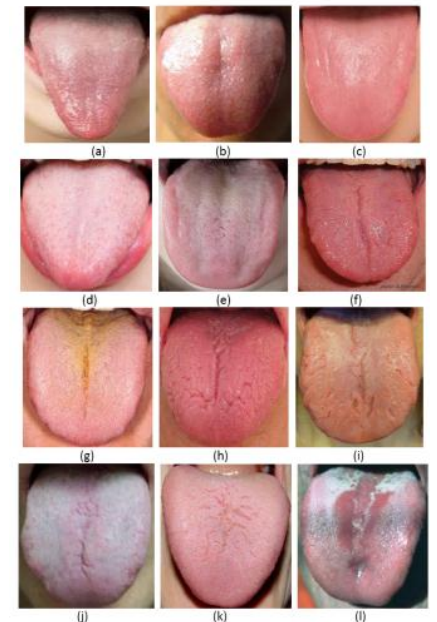

Figure-1 Images from internet
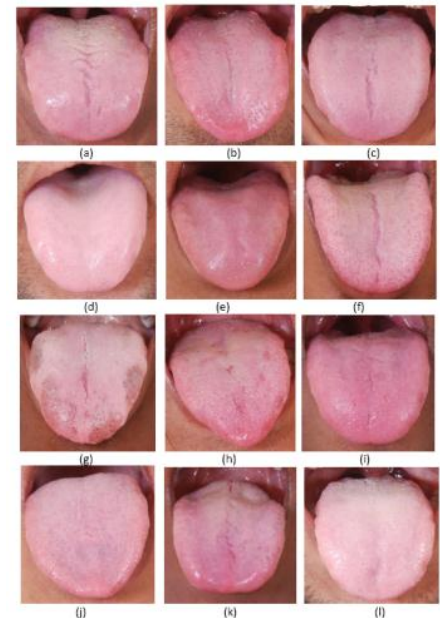

Figure-2 Images of students of JNTUH

The algorithm for the proposed scheme is given in Figure-3. It is implemented with two databases one after another. The two types of wavelets Haar and Daubechies are implemented separately with both of the databases. The values of ETF-1 and ETf-2 and TSF-1, TSF- 2 and TSF-3 are determined after performing number of iterations on the images of JNTUH database and with the help of Medical practitioner. 


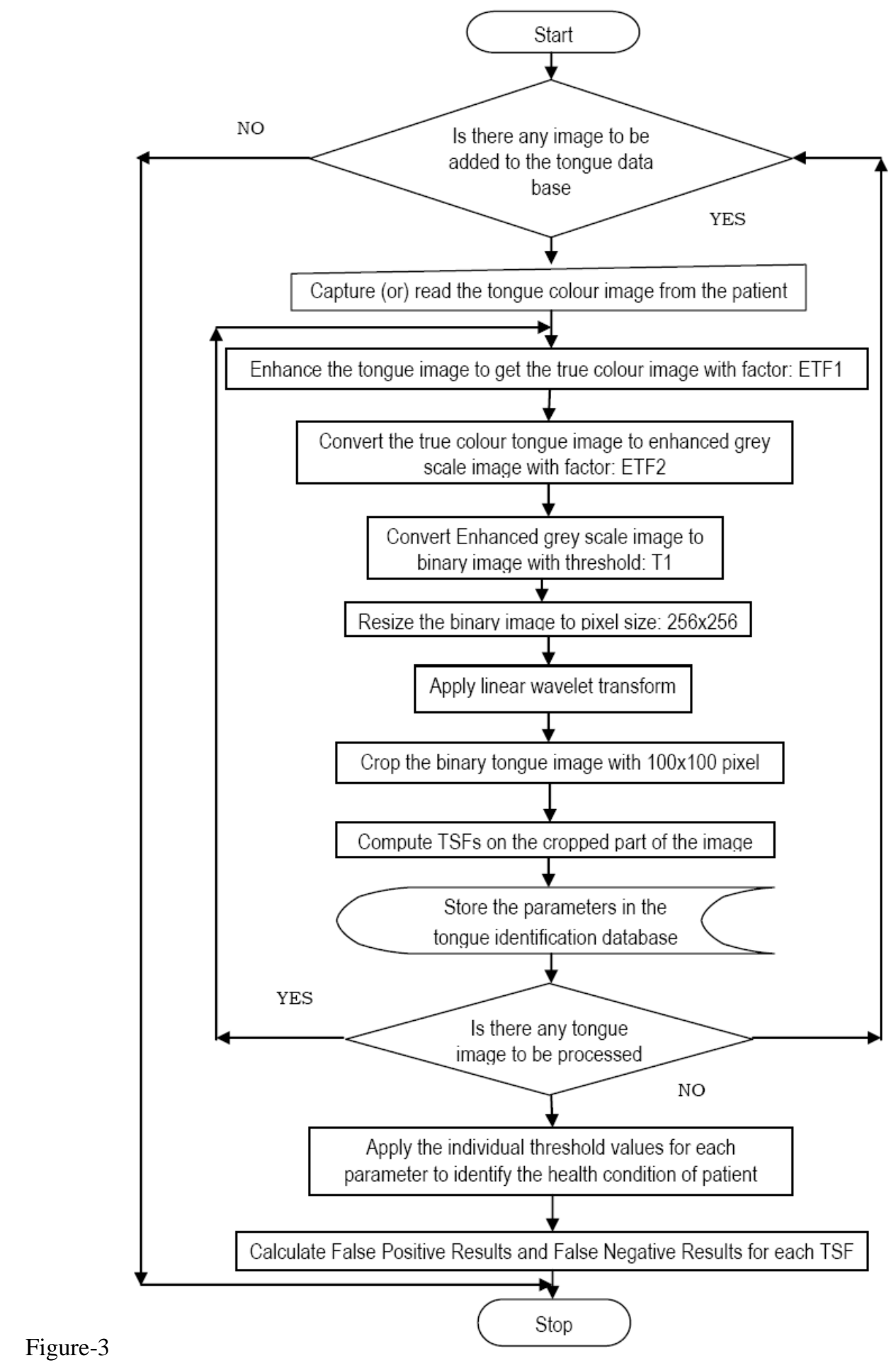

Figure-3

\section{RESULTS AND DISCUSSION}

The following figures and tables show the analysis results of the tongue statistical feature analysis data for the healthy and unhealthy persons after the implementation of the above Algorithm/Flowchart. The factors ETF-1, 2, T1 and TSF-1, 2, 3 are established after learning through evaluation of the results. The three statistical features are applied on 154 tongue images from JNTUH CEJ student's database. The tongue statistical features are evaluated on each of the tongue image as explained in above section and listed sample results in Table 1 and 3 with Enhanced Grey scale method and Enhanced True color method respectively.

The below tables show the example results of image analysis of the tongue after applying statistical measuring techniques on the wavelet transformed images. Tables- 1,2,3,4 belongs to JNTUH students database and Tables- 5,6,7,8 belongs to internet database. H/Uh indicates Healthy / Unhealthy condition of the person. 
Table-1

\begin{tabular}{|c|c|c|c|}
\hline \multicolumn{5}{|c|}{ EGS-Db6 wavelet transform -H/UH-JNTUH } \\
\hline $\begin{array}{c}\text { Image } \\
\text { Name }\end{array}$ & TSF1 & TSF2 & TSF3 \\
\hline T-2a-ju & 1.7931 & 0.5208 & 0.3013 \\
\hline T-2b-ju & 1.8104 & 0.4815 & 0.2381 \\
\hline T-2c-ju & 1.7858 & 0.5339 & 0.3199 \\
\hline T-2d-jh & 1.9722 & 0.2071 & 0.0668 \\
\hline T-2e-jh & 1.9917 & 0.1106 & 0.0207 \\
\hline T-2f-ju & 1.8367 & 0.4766 & 0.2658 \\
\hline T-2g-ju & 1.7858 & 0.5339 & 0.3199 \\
\hline T-2h-ju & 1.8254 & 0.4807 & 0.2579 \\
\hline T-2i-ju & 1.7927 & 0.5532 & 0.3690 \\
\hline T-2j-jh & 1.9891 & 0.1182 & 0.0170 \\
\hline T-2k-ju & 1.8024 & 0.4963 & 0.2579 \\
\hline T-2h-jh & 1.9903 & 0.1248 & 0.0293 \\
\hline
\end{tabular}

Table-3

\begin{tabular}{|c|c|c|c|}
\hline \multicolumn{4}{|c|}{ TC-Db6 wavelet transform -H/UH-JNTUH } \\
\hline $\begin{array}{c}\text { Image } \\
\text { Name }\end{array}$ & TSF1 & TSF2 & TSF3 \\
\hline T-2a-ju & 1.8439 & 0.4700 & 0.2627 \\
\hline T-2b-ju & 1.8019 & 0.5094 & 0.2876 \\
\hline T-2c-ju & 1.7815 & 0.5463 & 0.3415 \\
\hline T-2d-jh & 1.7146 & 0.5822 & 0.3548 \\
\hline T-2e-jh & 1.9848 & 0.1523 & 0.0387 \\
\hline T-2f-ju & 1.8058 & 0.5275 & 0.3298 \\
\hline T-2g-ju & 1.8802 & 0.4279 & 0.2357 \\
\hline T-2h-ju & 1.7631 & 0.5509 & 0.3308 \\
\hline T-2i-ju & 1.8099 & 0.5452 & 0.3720 \\
\hline T-2j-jh & 1.9989 & 0.0454 & 0.0058 \\
\hline T-2k-ju & 1.7973 & 0.5017 & 0.2627 \\
\hline T-2h-jh & 1.9752 & 0.1750 & 0.0274 \\
\hline
\end{tabular}

Table-5

\begin{tabular}{|c|c|c|c|}
\hline \multicolumn{4}{|c|}{ EGS-Db6 wavelet transform -H/UH-Google } \\
\hline $\begin{array}{c}\text { Image } \\
\text { Name }\end{array}$ & TSF1 & TSF2 & TSF3 \\
\hline T-1a-gh & 1.9909 & 0.1192 & 0.0259 \\
\hline T-1b-gh & 1.9871 & 0.1245 & 0.0152 \\
\hline T-1c-gh & 1.9730 & 0.2147 & 0.0803 \\
\hline T-1d-gh & 1.9579 & 0.2285 & 0.0526 \\
\hline T-1e-gh & 1.9970 & 0.0580 & 0.0027 \\
\hline T-1f-gu & 1.8225 & 0.4924 & 0.2787 \\
\hline T-1g-gu & 1.8090 & 0.5045 & 0.2855 \\
\hline T-1h-gu & 1.8661 & 0.4574 & 0.2710 \\
\hline T-1i-gu & 1.8548 & 0.4637 & 0.2666 \\
\hline T-1j-gu & 1.8752 & 0.4500 & 0.2710 \\
\hline T-1k-gu & 1.9193 & 0.3690 & 0.1991 \\
\hline T-11-gu & 1.8373 & 0.5298 & 0.3741 \\
\hline
\end{tabular}

Table-7

TC -Db6 wavelet transform -H/UH- Google

\begin{tabular}{|c|c|c|c|}
\hline $\begin{array}{c}\text { Image } \\
\text { Name }\end{array}$ & TSF1 & TSF2 & TSF3 \\
\hline T-1a-gh & 1.9963 & 0.0677 & 0.0058 \\
\hline T-1b-gh & 1.9734 & 0.1898 & 0.0440 \\
\hline T-1c-gh & 1.9438 & 0.2799 & 0.0977 \\
\hline T-1d-gh & 1.9943 & 0.0992 & 0.0214 \\
\hline T-1e-gh & 1.9389 & 0.2818 & 0.0875 \\
\hline T-1f-gu & 1.8914 & 0.4138 & 0.2293 \\
\hline T-1g-gu & 1.8724 & 0.4544 & 0.2758 \\
\hline T-1h-gu & 1.8664 & 0.4666 & 0.2905 \\
\hline T-1i-gu & 1.9038 & 0.3942 & 0.2140 \\
\hline T-1j-gu & 1.8914 & 0.4247 & 0.2504 \\
\hline T-1k-gu & 1.8140 & 0.5403 & 0.3668 \\
\hline T-11-gu & 1.8799 & 0.4345 & 0.2483 \\
\hline
\end{tabular}

Table-2

\begin{tabular}{|c|c|c|c|}
\hline \multicolumn{4}{|c|}{ EGS-Haar wavelet transform -H/UH-JNTUH } \\
\hline $\begin{array}{c}\text { Image } \\
\text { Name }\end{array}$ & TSF1 & TSF2 & TSF3 \\
\hline T-2a-ju & 0.8783 & 0.3270 & 0.5343 \\
\hline T-2b-ju & 0.9054 & 0.2927 & 0.4516 \\
\hline T-2c-ju & 0.8898 & 0.3132 & 0.5005 \\
\hline T-2d-jh & 0.9987 & 0.0366 & 0.0148 \\
\hline T-2e-jh & 0.9866 & 0.1152 & 0.1028 \\
\hline T-2f-ju & 0.8909 & 0.3118 & 0.4972 \\
\hline T-2g-ju & 0.8672 & 0.3394 & 0.5650 \\
\hline T-2h-ju & 0.9151 & 0.2788 & 0.4193 \\
\hline T-2i-ju & 0.9100 & 0.2863 & 0.4366 \\
\hline T-2j-jh & 0.9291 & 0.2568 & 0.3695 \\
\hline T-2k-ju & 0.8844 & 0.3197 & 0.5165 \\
\hline T-2h-jh & 0.9882 & 0.1081 & 0.0927 \\
\hline
\end{tabular}

Table-4

\begin{tabular}{|c|c|c|c|}
\hline \multicolumn{4}{|c|}{ TC-Haar wavelet transform -H/UH-JNTUH } \\
\hline $\begin{array}{c}\text { Image } \\
\text { Name }\end{array}$ & TSF1 & TSF2 & TSF3 \\
\hline T-2a-ju & 0.9159 & 0.2776 & 0.4165 \\
\hline T-2b-ju & 0.9250 & 0.2634 & 0.3842 \\
\hline T-2c-ju & 0.9288 & 0.2572 & 0.3705 \\
\hline T-2d-jh & 0.9984 & 0.0401 & 0.0173 \\
\hline T-2e-jh & 0.9801 & 0.1396 & 0.1408 \\
\hline T-2f-ju & 0.9059 & 0.2920 & 0.4499 \\
\hline T-2g-ju & 0.9282 & 0.2581 & 0.3724 \\
\hline T-2h-ju & 0.9191 & 0.2727 & 0.4053 \\
\hline T-2i-ju & 0.9323 & 0.2513 & 0.3574 \\
\hline T-2j-jh & 0.9895 & 0.1019 & 0.0840 \\
\hline T-2k-ju & 0.9180 & 0.2744 & 0.4091 \\
\hline T-2h-jh & 0.9626 & 0.1897 & 0.2300 \\
\hline
\end{tabular}

Table-6

\begin{tabular}{|c|c|c|c|}
\hline \multicolumn{3}{|c|}{ EGS-Haar wavelet transform -H/UH- Google } \\
\hline $\begin{array}{c}\text { Image } \\
\text { Name }\end{array}$ & TSF1 & TSF2 & TSF3 \\
\hline T-1a-gh & 0.9919 & 0.0894 & 0.0677 \\
\hline T-1b-gh & 0.9589 & 0.1986 & 0.2474 \\
\hline T-1c-gh & 0.9688 & 0.1738 & 0.2002 \\
\hline T-1d-gh & 0.9737 & 0.1602 & 0.1757 \\
\hline T-1e-gh & 0.9855 & 0.1196 & 0.1094 \\
\hline T-1f-gu & 0.9277 & 0.2590 & 0.3744 \\
\hline T-1g-gu & 0.9065 & 0.2912 & 0.4481 \\
\hline T-1h-gu & 0.9180 & 0.2744 & 0.4091 \\
\hline T-1i-gu & 0.8930 & 0.3091 & 0.4907 \\
\hline T-1j-gu & 0.9374 & 0.2423 & 0.3377 \\
\hline T-1k-gu & 0.9137 & 0.2808 & 0.4239 \\
\hline T-11-gu & 0.8965 & 0.3046 & 0.4799 \\
\hline
\end{tabular}

\section{Table-8}

\begin{tabular}{|c|c|c|c|}
\hline \multicolumn{3}{|c|}{ TC -Haar wavelet transform -H/UH- Google } \\
\hline $\begin{array}{c}\text { Image } \\
\text { Name }\end{array}$ & TSF1 & TSF2 & TSF3 \\
\hline T-1a-gh & 0.9788 & 0.1442 & 0.1483 \\
\hline T-1b-gh & 0.9890 & 0.1044 & 0.0875 \\
\hline T-1c-gh & 0.9645 & 0.1850 & 0.2211 \\
\hline T-1d-gh & 0.9729 & 0.1625 & 0.1799 \\
\hline T-1e-gh & 0.9965 & 0.0590 & 0.0335 \\
\hline T-1f-gu & 0.8987 & 0.3018 & 0.4732 \\
\hline T-1g-gu & 0.9231 & 0.2664 & 0.3910 \\
\hline T-1h-gu & 0.9398 & 0.2379 & 0.3282 \\
\hline T-1i-gu & 0.9782 & 0.1459 & 0.1513 \\
\hline T-1j-gu & 0.8925 & 0.3098 & 0.4923 \\
\hline T-1k-gu & 0.9153 & 0.2784 & 0.4184 \\
\hline T-11-gu & 0.9766 & 0.1511 & 0.1600 \\
\hline
\end{tabular}

The evaluation of False Negative and False Positive results for failure tongue identification of each technique on tongue database are given in Table 9, 10, 11 and 12. 
Applying Linear Wavelet Transforms and Statistical feature Analysis for Digital Tongue Image

Table-9 Rate of FPR and FNR for Db6 wavelet transform on internet database

\begin{tabular}{|c|c|c|c|c|c|}
\hline \multirow[b]{2}{*}{ Method } & \multirow[b]{2}{*}{ TSF } & \multirow[b]{2}{*}{ Threshold } & \multicolumn{3}{|c|}{ Db6 wavelet Transform } \\
\hline & & & $\%$ FPR & $\% \mathrm{FNR}$ & $\begin{array}{c}\text { Failure Rate of } \\
\text { Total Images }\end{array}$ \\
\hline \multirow{3}{*}{ EGS } & TSF1 & $\begin{array}{c}\text { If TSF1 }>1.94 \\
\text { Healthy else } \\
\text { Unhealthy }\end{array}$ & $14.70 \%$ & $10.54 \%$ & $11.03 \%$ \\
\hline & TSF2 & $\begin{array}{c}\text { If TSF } 2<0.4 \\
\text { Healthy else } \\
\text { Unhealthy }\end{array}$ & $11.76 \%$ & $12.10 \%$ & $12.06 \%$ \\
\hline & TSF3 & $\begin{array}{c}\text { If TSF }<0.2 \\
\text { Healthy else } \\
\text { Unhealthy }\end{array}$ & $17.64 \%$ & $10.93 \%$ & $11.72 \%$ \\
\hline \multirow{3}{*}{$\mathrm{TC}$} & TSF1 & $\begin{array}{l}\text { If TSF1 }>1.9 \\
\text { Healthy else } \\
\text { Unhealthy }\end{array}$ & $11.76 \%$ & $9.37 \%$ & $11.31 \%$ \\
\hline & TSF2 & $\begin{array}{c}\text { If TSF2 }<0.4 \\
\text { Healthy else } \\
\text { Unhealthy }\end{array}$ & $8.82 \%$ & $8.98 \%$ & $8.96 \%$ \\
\hline & TSF3 & $\begin{array}{c}\text { If TSF3 }<0.2 \\
\text { Healthy else } \\
\text { Unhealthy }\end{array}$ & $14.70 \%$ & $12.10 \%$ & $12.41 \%$ \\
\hline
\end{tabular}

Table-10 Rate of FPR and FNR for Haar wavelet transform on internet database

\begin{tabular}{|c|c|c|c|c|c|}
\hline \multirow[b]{2}{*}{ Method } & \multirow[b]{2}{*}{ TSF } & \multirow[b]{2}{*}{ Threshold } & \multicolumn{3}{|c|}{ Haar wavelet Transform } \\
\hline & & & $\% \mathrm{FPR}$ & $\%$ FNR & $\begin{array}{c}\text { Failure Rate of } \\
\text { Total Images }\end{array}$ \\
\hline \multirow{3}{*}{ EGS } & TSF1 & $\begin{array}{c}\text { If TSF1 }>0.96 \\
\text { Healthy else } \\
\text { Unhealthy }\end{array}$ & $17.64 \%$ & $12.50 \%$ & $13.10 \%$ \\
\hline & TSF2 & $\begin{array}{c}\text { If TSF } 2<0.2 \\
\text { Healthy else } \\
\text { Unhealthy }\end{array}$ & $14.71 \%$ & $13.67 \%$ & $13.79 \%$ \\
\hline & TSF3 & $\begin{array}{c}\text { If TSF }<0.25 \\
\text { Healthy else } \\
\text { Unhealthy }\end{array}$ & $20.58 \%$ & $11.32 \%$ & $12.41 \%$ \\
\hline \multirow{3}{*}{$\mathrm{TC}$} & TSF1 & $\begin{array}{c}\text { If TSF1 }>0.96 \\
\text { Healthy else } \\
\text { Unhealthy }\end{array}$ & $14.70 \%$ & $10.93 \%$ & $11.37 \%$ \\
\hline & TSF2 & $\begin{array}{l}\text { If TSF } 2<0.2 \\
\text { Healthy else } \\
\text { Unhealthy }\end{array}$ & $11.76 \%$ & $11.71 \%$ & $11.51 \%$ \\
\hline & TSF3 & $\begin{array}{l}\text { If TSF3 }<0.2 \\
\text { Healthy else } \\
\text { Unhealthy }\end{array}$ & $17.64 \%$ & $14.06 \%$ & $14.48 \%$ \\
\hline
\end{tabular}

EGS- enhanced grey scale method of image enhancement, TC- true colour method of image enhancement

Figure-4 FPR for internet database

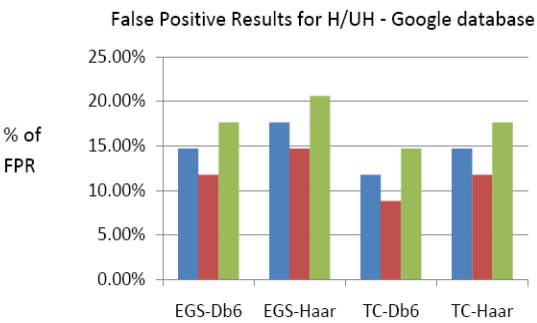

FPR - False positive results

Table-11 Rate of FPR and FNR for Db6 wavelet transform on JNTUH database

\begin{tabular}{|c|c|c|c|c|c|}
\hline \multirow[b]{2}{*}{ Method } & \multirow[b]{2}{*}{ TSF } & \multirow[b]{2}{*}{ Threshold } & \multicolumn{3}{|c|}{ Db6 wavelet Transform } \\
\hline & & & $\% \mathrm{FPR}$ & $\%$ FNR & $\begin{array}{c}\text { Failure Rate on } \\
\text { Total Images }\end{array}$ \\
\hline \multirow{3}{*}{ EGS } & TSF1 & $\begin{array}{c}\text { If TSF1 }>1.94 \\
\text { Healthy else } \\
\text { Unhealthy }\end{array}$ & $13.75 \%$ & $8.89 \%$ & $12.82 \%$ \\
\hline & TSF2 & $\begin{array}{c}\text { If TSF }<0.3 \\
\text { Healthy else } \\
\text { Unhealthy }\end{array}$ & $10.58 \%$ & $11.11 \%$ & $10.68 \%$ \\
\hline & TSF3 & $\begin{array}{c}\text { If TSF3 }<0.16 \\
\text { Healthy else } \\
\text { Unhealthy }\end{array}$ & $15.34 \%$ & $6.67 \%$ & $13.66 \%$ \\
\hline \multirow{3}{*}{$\mathrm{TC}$} & TSF1 & $\begin{array}{c}\text { If TSF1 }>1.85 \\
\text { Healthy else } \\
\text { Unhealthy }\end{array}$ & $11.11 \%$ & $8.89 \%$ & $10.68 \%$ \\
\hline & TSF2 & $\begin{array}{c}\text { If TSF }<0.3 \\
\text { Healthy else } \\
\text { Unhealthy }\end{array}$ & $7.94 \%$ & $6.67 \%$ & $7.69 \%$ \\
\hline & TSF3 & $\begin{array}{c}\text { If TSF3 }<0.25 \\
\text { Healthy else } \\
\text { Unhealthy }\end{array}$ & $13.23 \%$ & $11.11 \%$ & $12.82 \%$ \\
\hline
\end{tabular}

Figure-5 FNR for internet database

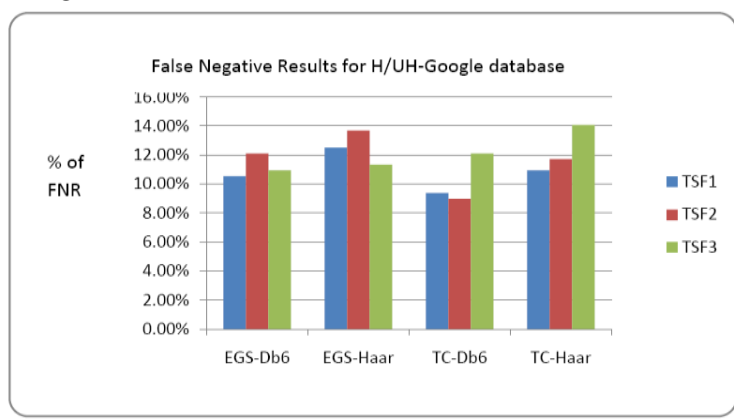

FNR - False negative results

Table-12 Rate of FPR and FNR for Haar wavelet transform on JNTUH database

\begin{tabular}{|c|c|c|c|c|c|}
\hline \multirow[b]{2}{*}{ Method } & \multirow[b]{2}{*}{ TSF } & \multirow[b]{2}{*}{ Threshold } & \multicolumn{3}{|c|}{ Haar wavelet Transform } \\
\hline & & & $\%$ FPR & $\%$ FNR & $\begin{array}{c}\text { Failure Rate on } \\
\text { Total Images }\end{array}$ \\
\hline \multirow{3}{*}{ EGS } & TSF1 & $\begin{array}{c}\text { If TSF1 }>0.94 \\
\text { Healthy else } \\
\text { Unhealthy }\end{array}$ & $16.40 \%$ & $8.89 \%$ & $14.96 \%$ \\
\hline & TSF2 & $\begin{array}{c}\text { If TSF2 }<0.25 \\
\text { Healthy else } \\
\text { Unhealthy }\end{array}$ & $14.28 \%$ & $11.11 \%$ & $13.68 \%$ \\
\hline & TSF3 & $\begin{array}{c}\text { If TSF3 }<0.3 \\
\text { Healthy else } \\
\text { Unhealthy }\end{array}$ & $18.52 \%$ & $8.89 \%$ & $16.67 \%$ \\
\hline \multirow{3}{*}{$\mathrm{TC}$} & TSF1 & $\begin{array}{c}\text { If TSF1 >0.95 } \\
\text { Healthy else } \\
\text { Unhealthy }\end{array}$ & $13.23 \%$ & $6.67 \%$ & $11.97 \%$ \\
\hline & TSF2 & $\begin{array}{c}\text { If TSF2 < } 0.2 \\
\text { Healthy else } \\
\text { Unhealthy }\end{array}$ & $10.05 \%$ & $11.11 \%$ & $10.26 \%$ \\
\hline & TSF3 & $\begin{array}{c}\text { If TSF3 }<0.2 \\
\text { Healthy else } \\
\text { Unhealthy }\end{array}$ & $15.34 \%$ & $13.33 \%$ & $14.96 \%$ \\
\hline
\end{tabular}


Figure-6 FPR for JNTUH database

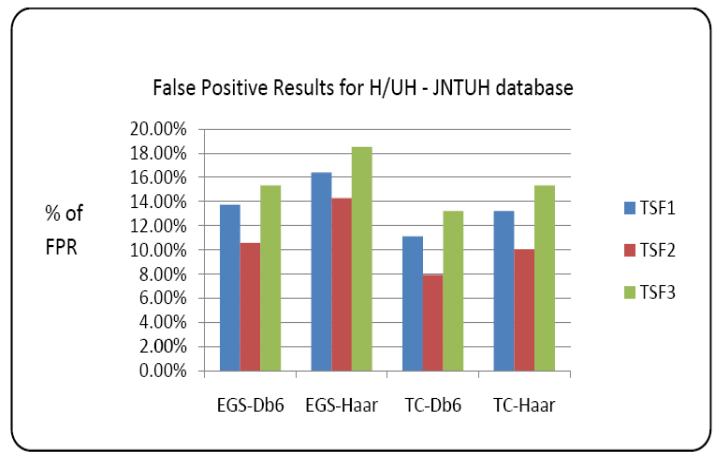

Figure-7 FNR for JNTUHdatabase

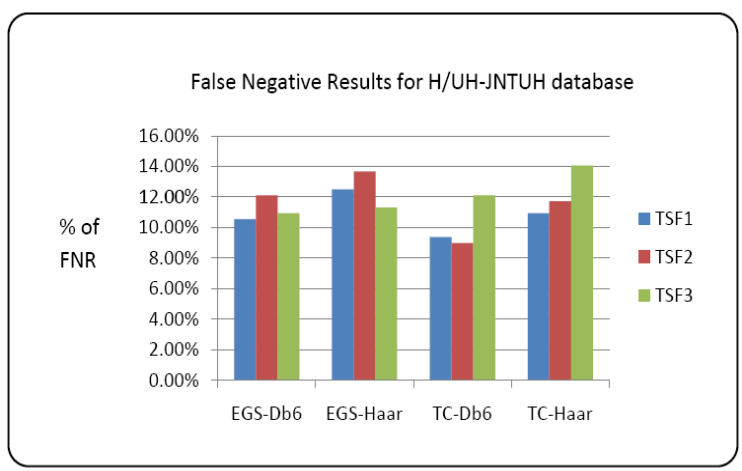

The success identification rate of healthy $\&$ un-healthy tongue images with enhanced grey scale $\&$ true color images are given in Table 13 and 14. Overall comparison is explained in the figure- 8.

Table-13 Success rates of Db6 and Haar transforms Table-14 Success rates of Db6 and Haar transforms $\mathrm{H} / \mathrm{UH}$ - internet database

\begin{tabular}{|c|c|c|c|}
\hline Method & TSF & $\begin{array}{c}\text { Success Rate with Db6 } \\
\text { wavelet transform }\end{array}$ & $\begin{array}{c}\text { Success Rate with } \\
\text { Haar wavelet } \\
\text { transform }\end{array}$ \\
\hline \multirow{3}{*}{ EGS } & TSF1 & $88.97 \%$ & $86.90 \%$ \\
\cline { 2 - 4 } & TSF2 & $87.94 \%$ & $86.21 \%$ \\
\cline { 2 - 4 } & TSF3 & $88.28 \%$ & $87.59 \%$ \\
\hline \multirow{3}{*}{ TC } & TSF1 & $88.69 \%$ & $88.63 \%$ \\
\cline { 2 - 4 } & TSF2 & $91.04 \%$ & $88.41 \%$ \\
\cline { 2 - 4 } & TSF3 & $87.59 \%$ & $85.52 \%$ \\
\hline
\end{tabular}

H/UH- JNTUH database

\begin{tabular}{|c|c|c|c|}
\hline \multirow{2}{*}{ Method } & TSF & $\begin{array}{c}\text { Success Rate with Db6 } \\
\text { wavelet transform }\end{array}$ & $\begin{array}{c}\text { Success Rate with } \\
\text { Haar wavelet } \\
\text { transform }\end{array}$ \\
\hline \multirow{3}{*}{ EGS } & TSF1 & $87.18 \%$ & $85.04 \%$ \\
\cline { 2 - 4 } & TSF2 & $89.32 \%$ & $86.32 \%$ \\
\cline { 2 - 4 } & TSF3 & $86.34 \%$ & $83.33 \%$ \\
\hline \multirow{3}{*}{ TC } & TSF1 & $89.32 \%$ & $88.03 \%$ \\
\cline { 2 - 4 } & TSF2 & $92.31 \%$ & $89.74 \%$ \\
\cline { 2 - 4 } & TSF3 & $87.18 \%$ & $85.04 \%$ \\
\hline
\end{tabular}

Figure-8 Comparison of H/UH success rate on JNTUH and Internet databases using Db6 and Haar wavelet transforms

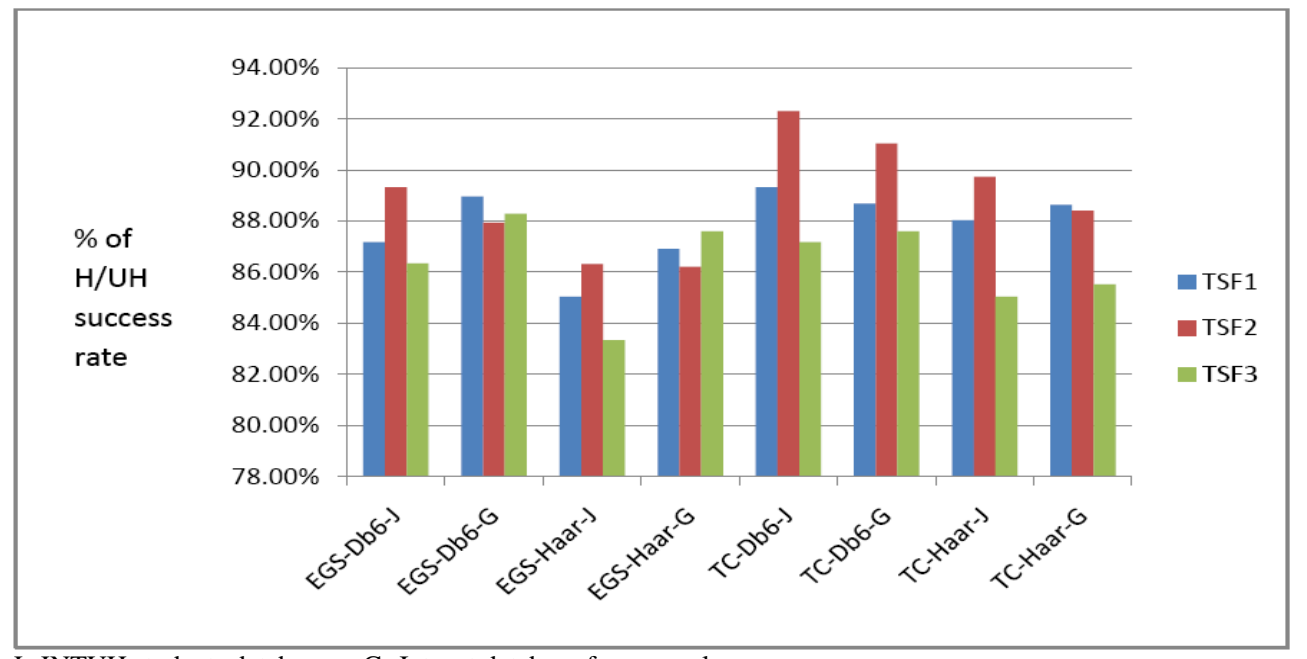

J- JNTUH students database, G- Intenet database from google

\section{CONCLUSION}

Tongue image analysis is one of the useful non invasive diagnostic tools for Ayurvedic medical practitioner. In this paper a novel method for Tongue analysis based one-level linear wavelet decomposed texture features derived from co-occurrence matrices in all orientation. Thus, in current method, enhancement is done by finding suitable threshold values which are Tongue Enhancement Factors to reproduce true color image and white coating of the tongue by color image processing and cracks and pimples/buds were highlighted by processing the image in grey scale. In the proposed method the tongue statistical features TSF1 and TSF2 are 
having better performance than TSF3. Applying enhanced True color technique, an average of $92 \%$ of Healthy and Un-healthy tongue images are identified correctly. Among the two wavelet decomposition techniques that are used, the Daubechies is having slightly better performance than Haar. On over all basis it is proven that the performance (speed, recognition rate) of the Tongue analysis system is improved by applying one-level linear wavelet decomposition techniques. From the evaluation of the results it is showed that the proposed methods gives the appropriate results and is well suited for the tongue image processing. Further enhancement to the system can be done by improving the localized intensity methods and calculation of the features and giving an empirical table to identify the tongue disease wise. And also the performance of this system is more for standard tongue database than images down loaded from Google Images. This variation is due to the changes in image capturing environment, noise, and variation in properties of the images that are down loaded from Google Images.

\section{Acknowledgements}

The authors would like to express their cordial thanks to Dr. Rameshwar rao, Honble' Vice chancellor, JNTUH, and Dr.Tirumalachary, Principal, College of Engineering- CEJ-Karimnagar for permitting me to do this research work. Authors would like to acknowledge the support of Dr. Santosh Kumari, BAMS, for her help in making us understand the tongue and her cooperation in the analysis of tongue images.

\section{REFERENCES}

[1] 'Nutrition Central, informed, involved and inspired' by: Edward Thompson, Issue: Autumn, Year of publication by Institute of Optimum Nutrition: 2001

[2] 'Introduction About Traditional Tongue Diagnosis With Scientific Value Addition', Dr. Debasis Bakshi, Dr Sujata Pal, Proceedings of 2010 International Conference on Systems in Medicine and Biology, 16-18 December 2010, IIT Kharagpur, India

[3] 'Ayurvedic Tongue Diagnosis' by Walter' Shantree 'Kasera, Eddition 2007, Mothilal Banarsidass publishers, No. 3, June 2012.

[4] Wangmeng Zuo, Kuanquan Wang, David Zhang, and Hongzhi Zhang, "Combination of Polar Edge Detection and Active Contour Model for Automated Tongue Segmentation", In Proceedings of the Third International Conference on Image and Graphics, pp: 270-273, 2004

[5] Bo Pang, David Zhang, Kuanquan Wang, "Tongue image analysis forappendicitis diagnosis", Transactions on Information Sciences, Vol. 175, No. 3, pp: 169-176, 2005.

[6] Yang Ben Sheng, Wei Yu Ke, Li Jiang Ping, "Research and Application of Image Segmentation Algorithm Based on the Shortest Path in Medical Tongue Processing”, WRI World Congress on Software Engineering, pp: 239-243, 2009.

[7] Wang X, Zhang D, “An Optimized Tongue Image Color Correction Scheme”, Ieee Transactions On Technol Biomed, Vol. 14, No. 6, 2010.

[8] Wenshu Li, Jianfu Yao, Linlin Yuan And Qinian Zhou, “The Segmentation Of The Body Of Tongue Based On The Improved Level Set In Tcm”, Life System Modeling And Intelligent Computing, Vol. 6330, Pp: 220-229, 2010.

[9] 'A New Pattern Recognition Algorithm And Its Application About Tongue Fur Image Classification Of Traditional Chinese Medicine' By Yuke Wei , Jiangping Li,Qun Chen , Mei Liu, 3rd International Conference on Biomedical Engineering and Informatics (BMEI 2010)

[10] 'An Optimized Tongue Image Color Correction Scheme', By Xing zheng Wang and David Zhang IEEE Transactions on Information Technology in Biomedicine, Vol. 14, no. 6, November 2010.

[11] 'Analysis of the Tongue Fur and Tongue Features by Naive Bayesian Classifier', By Weitong Huang, Zhaoqian Yan ,JiatuoXu, Li Zhang 2010 International Conference on Computer Application and System Modeling (ICCASM 2010).

[12] 'Automatic Tongue Diagnosis System', By 1Lun-chien Lo, 1Mark Chun-cheng Hou, 1Ying-ling Chen, 2John Y. Chiang, 2Chengchun Hs, 978-1-4244-4134-1/09/\$25.00 @2009 IEEE.

[13] 'Quantified Vector Oriented Tongue Color Classification', By Bo Huang, Kuanquan Wang, Xiangqian Wu, Dongyu Zhang and Naimin Li, 978-1-4244-4134-1/09/\$25.00 @2009 IEEE.

[14] 'Tongue Color Visualization for Local Pixel', By Bo Huang, David Zhang, 2011 3rd 1nternational Conference on Advanced Computer Control (ICACC 2011).

[15] 'Using Threshold Method to Separate the Edge, Coating and Body of Tongue in Automatic Tongue Diagnosis', By C. C. Wei $\backslash$ c. H. Wang \s. w. Huang2,.

[16] M. Antonini, M. Barlaud, P. Mathieu, I. Daubechies, Image coding using wavelet transform. IEEE Trans. Image Process. 1 (2), 205-220, 1992.

[17] 'The Haar-Wavelet Transform in Digital Image Processing:Its Status and Achievements', by Piotr Porwik, Agnieszka LisowskaMachine GRAPHICS \& VISION vol .13, n o. 1/2, 2004, p p .79-98

[18] I. Daubechies, Ten Lectures on Wavelets, Rutgers University and AT\&T Laboratories, 1992. [10]

[19] 'Importance of Statistical Measures in Digital Image Processing', By Vijay Kumar1, Priyanka Gupta2 , International Journal of Emerging Technology and Advanced Engineering (ISSN 2250-2459, Volume 2, Issue 8, August 2012). 